\title{
Implementation of Information Integration Platform in Chinese Tobacco Industry Enterprise Based on SOA
}

\author{
Hong-Iv Wang, Yong Cen \\ Information Center, China Tobacco Zhejiang Industrial Co., Ltd \\ Hangzhou, China, 310009 \\ wanghl@zjtobacco.com,ceny@zjtobacco.com
}

\begin{abstract}
Keywords: Information Integration; Tobacco Industry Enterprise; Service-Oriented-Architecture (SOA)
\end{abstract}

\begin{abstract}
An SOA-based information integration platform in Chinese tobacco industry enterprise was constituted with SAP XI. SAP XI as the ESB transforms the formats of all data coming from source systems to realize the seamless integration among different systems. The service bus of SAP XI links different systems in enterprises and factories and realizes the seamless integration among multiple business systems of different levels. The application of information integration platform ensures the flexible and controllable information communication among application systems in enterprise at architectural level and solves the problems of information island, data quality and flow integration.
\end{abstract}

\section{Introduction}

With the deepening of the tobacco industry information construction and development, "Information technology to promote industrialization" becomes the inevitable choice of the tobacco industry enterprises to improve production and management level. More and more information systems such as Enterprise Resource Planning (ERP), Product Data Management (PDM), Comprehensive Marketing Business System (CMB), Manufacturing Execution System (MES), Cigarette Industry Production and Management Decision Support System (CIPMDS), Digital Warehouse Management System (DWMS), and Corporate Economic Operation Analysis System (CEOAS),etc. have been constructed, implemented and put into use in tobacco industry enterprises, better support rapid development of the enterprise business needs [1]. However, these various kinds of information systems developed by different suppliers at various times, put into use for enterprise's information work have brought new problems. Mainly means the emergence of the problems about "Information Islands ", "Data Quality", and "Process Integration". It is the existence of these problems that affect the process of the tobacco industry informatization. Therefore, the tobacco industry enterprise after years of information construction, basically have undergone the control stage, preparation or preliminary into the integrated stage. In recent years, the tobacco industry enterprises to try and practice gradually increase in the integration of information [2], Most of them concentrated in the construction of the data asynchronous interaction, but the application of data interactive platform based on in real-time interactive services was less. Therefore, how to effectively integrate many business management information system, and effective integration of the existing information resources in tobacco industry enterprise, has become an important issue for the tobacco industry enterprise information construction work. Therefore, integration of informatization resources and application system is the focus of the present work and it is also the focus of this paper.

\section{Overview of SOA}

The Concept of SOA. At present, service oriented architecture (SOA) is one of the most popular topics which is an architecture building method used to describe, link and integrate the reusable business services with clear boundary and self-contained functions [3]. With its special loose 
coupling, coarse granularity, reusability and interoperability, SOA has become the effective method of information resources integration management, and is the important development direction of realizing the fusion of tobacco industry information and IT industry. SOA is an IT architecture style and is service-oriented. It is a new project philosophy characterized by modularity, separation of concerns, service re-uses, and composition, as well as a new program method based on standards and tools that largely involve web services [4].

Architecture Model of SOA. The essence of SOA is to integrate reusable services with distinct boundary and self-contained functions by the centralized management platform. The platform can link different services through the interfaces and the contracts defined among application services [5]. The SOA architecture can be classified as stratified structure. With several levels from the bottom functional service to atom service and service component, then to the top level business process service, it has one basis aim that do its terminal to encapsulate different kinds of service to make it as multiplex, and the same core-service of whichever level. In particular, a complex service component consists of different atom services, to provide functions needed in the process of business progress [6]. The architecture model of SOA has been given in Figure 1.

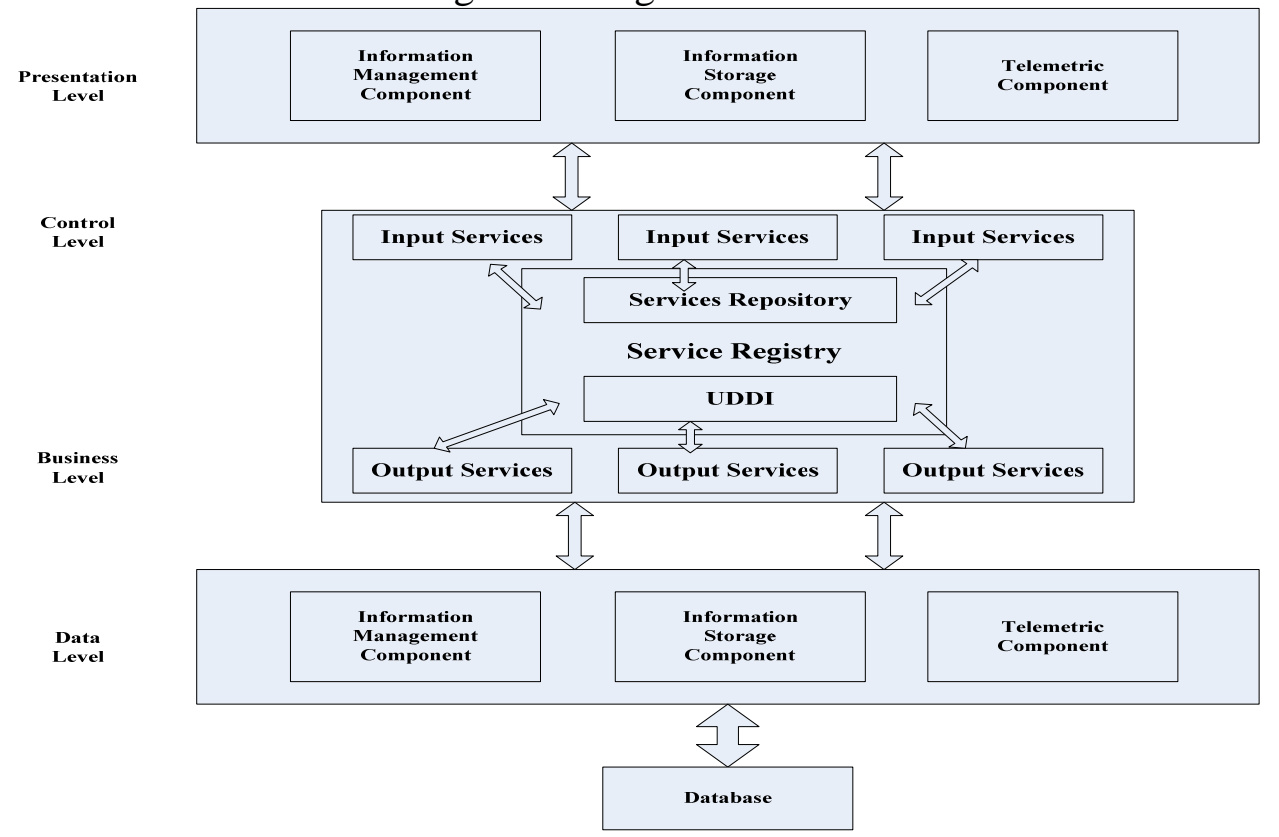

Fig.1 The architecture model of SOA

Implementation Technology of SOA. Currently, SOA is mainly realized in form of web services. Web services are based on XML (Extensive Makeup Language) and support the communication between different operating systems over a network with different program languages [7]. Web services are made up of four parts: XML, SOAP (Simple Object Access Protocol), WSDL (Web Services Description Language) and UDDI (Universal Description Discovery and Integration). XML is used to package the data, SOAP is used to transfer the data, describing the services available is used WSDL and listing what services are available is used UDDI. The service consumer can search the UDDI for the service's needs. Once the service consumer finds the required services, sends a request to the service provider asking for access to those services. The service consumer and provider exchange request/response messages (XML format) through the use of protocols such as SOAP.

\section{The Concept and Structure of SAP XI}

SAP XI (Exchange Infrastructure) for application integration platform as part of the SAP NetWeaver middleware products, which purpose is to provide a management information system data and processes interact with the SAP system platform for non-SAP systems.

SAP XI platform consists of two parts, the first part is called Integration Broker, which in the manner of the message exchange of data between systems (containing the two parts of the Adapter engine and Integration engine); another part is BPA (Business Processing Engine, BP Engine), 
business process automation, to achieve convergence of peripheral systems business flow and SAP business. These two parts are the two levels of functionality and BPA based on Integration Broker [8]. SAP XI is one of the core components of the SAP NetWeaver; it consolidated and developed middleware and BC (Business Connector) two kinds of conjunction.

SAP XI technology architecture show as the Figure 2, Integration Repository (IR) for scenario analysis and system design; Integration Directory (ID) for SAP XI object configuration and management; System Landscape Directory (SLD) for the conservation and management of the system examples created for the scenario; Integration Server for the message type, logic routing rules build and routing rules determine ABAP Proxy call selection and design.

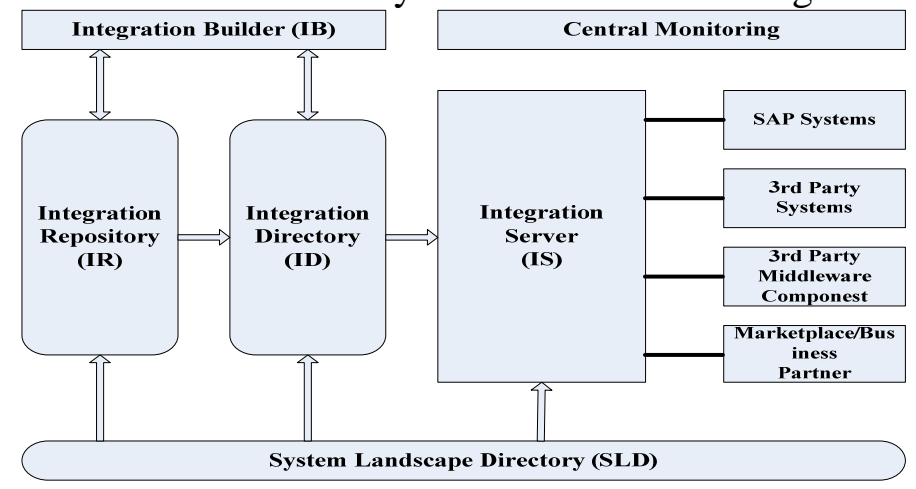

Fig.2 SAP XI technology architecture

\section{Information Integration Architecture Based on SAP XI}

Information Integration Architecture Design. Tobacco industry enterprise has very complicated business logic, numerous different systems, and high requirements of users. These features make the information integration of tobacco industry enterprise very difficult. According to the thought of loose coupling, SOA can ensure the flexibility, as well as control the complexity. So building an Information Integration Platform based on SOA is the best choice.

The core of information integration platform is the construction of Enterprise Service Bus (ESB) and unified service registry management system. Therefore, based on the construction of the status quo of enterprise application system and combined with features of SAP XI, use the SAP XI as the enterprise service bus (ESB), through the SOAP protocol and star-shape structure to realize the integration of various heterogeneous system. After each application system present situation research, demand discussion and blueprint design, the formation of the final solving strategies based on the overall system integration of SAP XI, show as Figure 3.

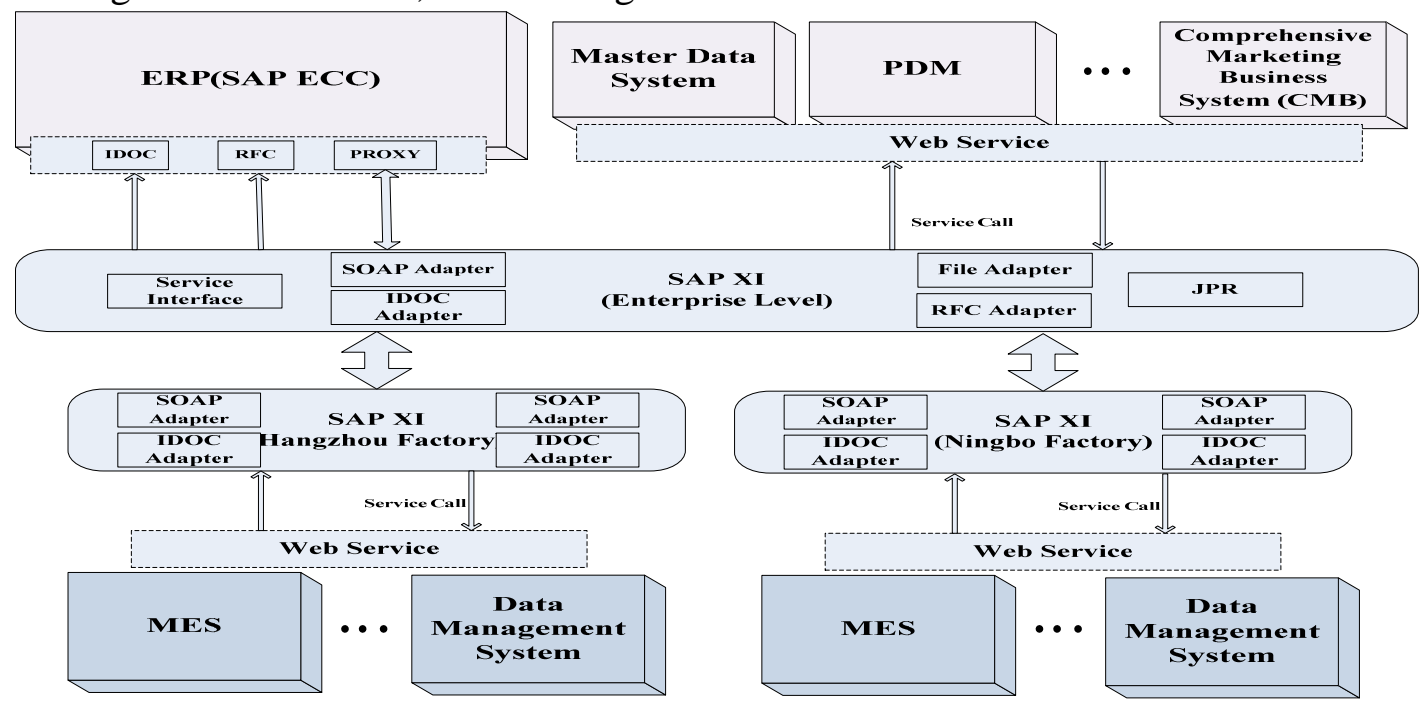

Fig.3 The overall system integration of SAP XI 
In the technical implementation, established star-shape connection with SAP XI as a central Hub, systems simply can make a connection with the SAP XI. In order to effectively avoid the sharp rise of complexity caused by the one-to-one connection between the middleware, system and system interconnect system integration [8]. In addition, SAP XI will convert all format of the data from the source system into an XML format for processing, determine its target system and then converted data into the target system required by the XML format for output, therefore, it can realize effective communication between heterogeneous systems integration.

SAP XI has played the role of an intermediary layer in the interaction of business systems, convergence between different information systems in the National Council, provincial companies, factories, to achieve a seamless integration between the different levels of multiple business systems, and presented to the overall state. Web Service to show different information resources provided by the service, register and exchange in SAP XI platform. When need for the service interaction between system, first of all the service requests sent to SAP XI exchange service bus platform, service bus is according to the source of the request and the context information query, unified registration management system get the Web service address and interface specification. According to the service security requirement, through the user directory for unified security authentication, then the service request routing forward to provide service business system for processing. Business system processing returns a response message to the service bus, service bus will be returned in response to the service requesting party.

Implementation Steps. Based on the SAP XI information integrated construction in general can be divided into the following three steps:

(1) Analysis Requirements. To analyze the existing between the system and need to existing interactive process and data needs.

(2) Establish standards. To analyze data need for information exchange between the various systems, and finishing form data standards, and carding sure interactive way. For batch non real-time requirements of the information interaction, use the already mature data exchange way to realization. For the real-time requirements of information interaction, use the service mode to realization, create the service directory list, then reference to the tobacco industry data exchange service system for Web services standards to description and definition of the specific interface content.

(3) Technology realization. For the above work is done, configured and defined in SAP XI, while the system interface development work completed in accordance with the standard specification.

Color figures are welcome for the online version of the journal. Generally, these figures will be reduced to black and white for the print version. The author should indicate on the checklist if he wishes to have them printed in full color and make the necessary payments in advance.

\section{Summary}

At present, tobacco industry enterprise will shift the focus of information construction to the integration of information resources and application systems. The goal is to achieve system integration, resource integration, information sharing in order to eliminate the "Information Island" and promote the development of information construction in tobacco industry enterprises. The technical characteristics of SOA determine it's a good selection for realizing above goal. In this paper, we mainly introduce the design of tobacco industry enterprise's Information integration Platform which based on service-oriented architecture and SAP XI technology. Based on the platform, it solves the software reuse and integration expansion problem in a distributed environment using a new way, that not only improves the efficiency of software development, but also enhance the information system of flexibility, reusability and evolutionary in the enterprise.

\section{References}


[1] Wei-Min Wang. Study and Application of Production-Marketing Integrated System of Group Governance in Tobacco Industry [J]. Tobacco Science \& Technology, 2007(5):29-33. (in Chinese).

[2] Xiao-Ying Li. Integration of Discrete Information Systems in Manufacturing Industry Based on SOA [J]. Tobacco Science \& Technology, 2010(6):41-44. (in Chinese).

[3] Hua-dong Wang, Qiang Hu. Research and Application of an Integration Platform for E-Commerce System Based on SOA[C]. International Conference on Management of E-commerce and E-government, 2009:424-427.

[4] Xiong-Yi Li. Research and Application of SOA in B2B Electronic Commerce[C]. International Conference on Computer Technology and Development, 2009: 649-653.

[5] Duan Yan-e. Research about Based-SOA Agriculture Management Information System[C]. International Conference on Information and Automation, 2012: 78-82.

[6] Huan-huan Zhuang, GAO Zhen, Xi-bo Zhu. Research on SOA-based Integration Solution to Bank Online Application System [C]. 2011 International Conference on Computer Science and Network Technology, 2011: 2235-2240.

[7] Kuang-Yu Peng, Shao-Chen Lui, Ming-Tsung Chen. A Study of Design and Implementation on SOA Governance: A Service Oriented Monitoring and Alarming Perspective. IEEE International Symposium on Service-Oriented System Engineering, 2008: 215-220.

[8] John Wiley, Sons. SAP NetWeaver For Dummies [M]. Wiley Publishing, 2005. 